\title{
Photoinduced changes in the cuprate electronic structure revealed by femtosecond time- and angle-resolved photoemission
}

\author{
J. D. Rameau, ${ }^{1}$ S. Freutel, ${ }^{2}$ L. Rettig,,${ }^{2,}{ }^{*}$ I. Avigo, ${ }^{2}$ M. Ligges,${ }^{2}$ Y. Yoshida,${ }^{3}$ H. Eisaki, ${ }^{3}$ J. Schneeloch, ${ }^{1}$ R. D. Zhong, ${ }^{1}$ \\ Z. J. Xu, ${ }^{1}$ G. D. Gu, ${ }^{1}$ P. D. Johnson, ${ }^{1}$ and U. Bovensiepen ${ }^{2}$ \\ ${ }^{1}$ Brookhaven National Laboratory, Upton, New York 11973, USA \\ ${ }^{2}$ Fakultät für Physik und Zentrum für Nanointegration Duisburg-Essen, Universität Duisburg-Essen, \\ Lotharstrasse 1, 47048 Duisburg, Germany \\ ${ }^{3}$ National Institute of Advanced Industrial Science and Technology, Tsukuba, Ibaraki 305-8568, Japan \\ (Received 20 September 2013; revised manuscript received 22 February 2014; published 13 March 2014)
}

\begin{abstract}
The dressing of quasiparticles in solids is investigated by changes in the electronic structure $E(\boldsymbol{k})$ driven by femtosecond laser pulses. Employing time- and angle-resolved photoemission on an optimally doped cuprate above $T_{\mathrm{c}}$, we observe two effects with different characteristic temporal evolutions and, therefore, different microscopic origins. First, a marked change in the effective mass due to the $70-\mathrm{meV}$ kink in $E(\boldsymbol{k})$ is found to occur during the experiment's 100-fs temporal resolution and is assigned to laser-driven perturbation of an electronic interaction dressing the bare band. Second, a change in $k_{\mathrm{F}}$ is explained by effective photodoping due to particle-hole asymmetry and offers opportunities for ultrafast optoelectronic switches based on an optically driven insulator-superconductor transition.
\end{abstract}

DOI: 10.1103/PhysRevB.89.115115

PACS number(s): 74.25.Jb, 74.72.Kf, 78.47.J-, 79.60.-i

\section{INTRODUCTION}

The nature of single-particle excitations and their interaction with collective phenomena have continued to present challenges in solid-state physics. A representative example of this problem can be found in the cuprates. The well-known 70meV "kink" in the electronic structure $E(\boldsymbol{k})$ and the associated mass renormalization [1] has elicited considerable controversy with phonons [2-4], spin excitations [5-7], and, indeed, more exotic mechanisms [8] invoked for its origin. One underlying problem in solving this issue is that the energy scales associated with these differing collective modes fall within a similar energy range, thereby, making a clear distinction nontrivial.

Recent developments in the field of femtosecond timeresolved spectroscopy [9] have opened new opportunities to analyze materials in a nonequilibrium state after optical excitation by a pump laser and to determine their characteristic responses and subsequent relaxation times to such external stimuli. In condensed matter, this dynamic response is dominated by coupling of single-particle excitations to further degrees of freedom in their environment, i.e., to other electrons and collective excitations. Pump-probe experiments reveal this response directly in the time domain and can, thereby, provide information not available to static spectroscopic probes.

The strong local interactions in the cuprates, with their pronounced screening of local interactions, appear as particularly promising candidates for such studies. Previous optical and terahertz pump-probe experiments have established the time scales at which pump-induced changes occur [10-14]. Below the critical temperature, for the onset of superconductivity, $T_{\mathrm{c}}$, recovery of the photoinduced perturbation of the superconducting condensate is found to proceed on a picosecond time scale. Above $T_{\mathrm{c}}$, the characteristic time scales are clearly faster and reach well into the femtosecond

\footnotetext{
*Present address: Swiss Light Source, Paul Scherrer Institute, 5232 Villigen PSI, Switzerland.
}

regime [10]. More recently, femtosecond time- and angleresolved photoemission spectroscopy (tr-ARPES) has added information on momentum-dependent dynamics [15-17].

Here, we use pump-probe techniques to offer new insights into the origin of this mass renormalization and to quantify femtosecond photoinduced doping by exploring interactions directly in the time domain. In particular, we employ tr-ARPES to investigate ultrafast changes in the occupied electronic structure $E(\boldsymbol{k})$ of optimally doped $\mathrm{Bi}_{2} \mathrm{Sr}_{2} \mathrm{CaCu}_{2} \mathrm{O}_{8+\delta}$ above $T_{\mathrm{c}}$. Pumping with IR light, we observe that the interaction producing the kink can be disrupted and recovers on time scales on the order of a few tens of femtoseconds. Furthermore, we present evidence that such pumping can result in an effective photodoping, quantified through a time-dependent shift in the Fermi momentum $k_{\mathrm{F}}$, pointing the way to the possibility of new femtosecond optoelectronic device technologies.

\section{EXPERIMENT}

Our experimental setup at the University of DuisburgEssen combines a commercial amplified Ti:sapphire laser system (Coherent RegA 9040) and a homebuilt time-of-flight electron spectrometer employing a position-sensitive delay line anode (Roentdek Hexanode). With this unique instrument, we measure the photoelectron intensity as a function of kinetic energy, two independent in-plane momentum directions $k_{x}$ and $k_{y}$, and pump-probe delay $t$ [18]. Time-resolved experiments were performed in a pump-probe geometry by first exciting the samples using the fundamental $p$-polarized (perpendicular to the nodal line) output of our laser system ( $h v_{\text {pump }}=$ $1.5 \mathrm{eV}, 40 \mathrm{fs}$ ) and subsequently probing the pump-induced dynamics by direct photoemission using suitably delayed frequency-quadrupled $s$-polarized (parallel to the nodal line) pulses $\left(h v_{\text {probe }}=6.0 \mathrm{eV}, 90 \mathrm{fs}\right)$. The fundamental and frequency-quadrupled pulse trains at a $250-\mathrm{kHz}$ repetition rate were focused on the sample to $(100 \mu \mathrm{m})^{2}$ and $(50 \mu \mathrm{m})^{2}$, respectively. The overall temporal, spectral, and momentum resolutions of the experiment were determined to be $100 \mathrm{fs}$, 
$55 \mathrm{meV}$, and $2 \times 10^{-3} \AA^{-1}$, respectively. The temporal overlap between pump and probe pulses was determined with an accuracy of $\Delta t_{0}= \pm 5$ fs by analyzing hot carrier relaxation as a function of time delay in $\mathrm{Bi}_{2} \mathrm{Sr}_{2} \mathrm{CaCu}_{2} \mathrm{O}_{8+\delta}$ in energy windows $E-E_{\mathrm{F}}=1.4-1.5 \mathrm{eV}$ above the Fermi level $E_{\mathrm{F}}$. The subsequent population dynamics showed negligible hot carrier lifetime contributions, thus, directly reflecting the pump-probe cross correlation (XC) which quantifies the temporal resolution, found to be $100 \pm 5 \mathrm{fs}$.

Single-crystal samples of $\mathrm{Bi}_{2} \mathrm{Sr}_{2} \mathrm{CaCu}_{2} \mathrm{O}_{8+\delta}$ grown using standard floating-zone furnace techniques were cleaved in situ under ultrahigh-vacuum conditions $\left(p<10^{-10} \mathrm{mbar}\right)$ at base temperatures of $100 \mathrm{~K}$. Photoemission spectra were recorded as four-dimensional data sets $I\left(E, k_{\mathrm{x}}, k_{\mathrm{y}}, t\right)$ with exposure times of $60 \mathrm{~s}$ in 20 subsequent scans. The probe-pulse intensity was carefully tuned to prevent spectral broadening due to space-charge effects, resulting in typical electron count rates of $100 \mathrm{kHz}$ at the electron analyzer. The Fermi energy $E_{\mathrm{F}}$ was referenced at $T=100 \pm 5 \mathrm{~K}$ to a 2.8 -nm-thick $\mathrm{Pb}$ film evaporated onto the samples using a calibrated Knudsen cell at the end of the measurement. Fitting of the FermiDirac distribution yielded a Gaussian energy resolution of $\Delta E=55 \pm 5 \mathrm{meV}$ full width at half maximum as confirmed separately by the probe beam's optical spectrum.

\section{RESULTS AND DISCUSSION}

Figures 1(a)-1(c) show the observed photoelectron intensity $I(E, k, t)$ along the nodal direction $(0,0)$ to $(\pi, \pi)$ for several $t$ 's. Aside from pronounced intensity changes, two more subtle, but essential, effects provide new insights into the excitations in these materials. As we show here, these effects include: (i) a rigid shift in the electronic band towards negative $k$ including a change in $k_{\mathrm{F}}$ and (ii) a change in the slope of the band near $E_{\mathrm{F}}$ implying a change in the effective mass $m^{*}$. The latter effect is observable both as a dynamic change in the low-energy dispersion in Figs. 2(e)-2(h) and in the causally related broadening of the momentum distribution curve (MDC) widths $\Delta k(E, t)$ below the kink energy shown in Fig. 1(e). To highlight these rather subtle changes, we have applied a Lucy-Richardson deconvolution algorithm to the data [19] where the Gaussian spectral broadening was used as input for the point-spread function in the deconvolution algorithm [20]. The $k_{\mathrm{F}}$ change is evident in the MDCs in Fig. 1(d), which show the intensity at $E_{\mathrm{F}}$ before and during pumping. The variation in the intensity maximum as a function of $k$ determines the $k_{\mathrm{F}}$ shift, discussed in more detail below.

The spectral weight redistribution driven by the optical excitation is depicted in Figs. 2(a)-2(d) where intensity
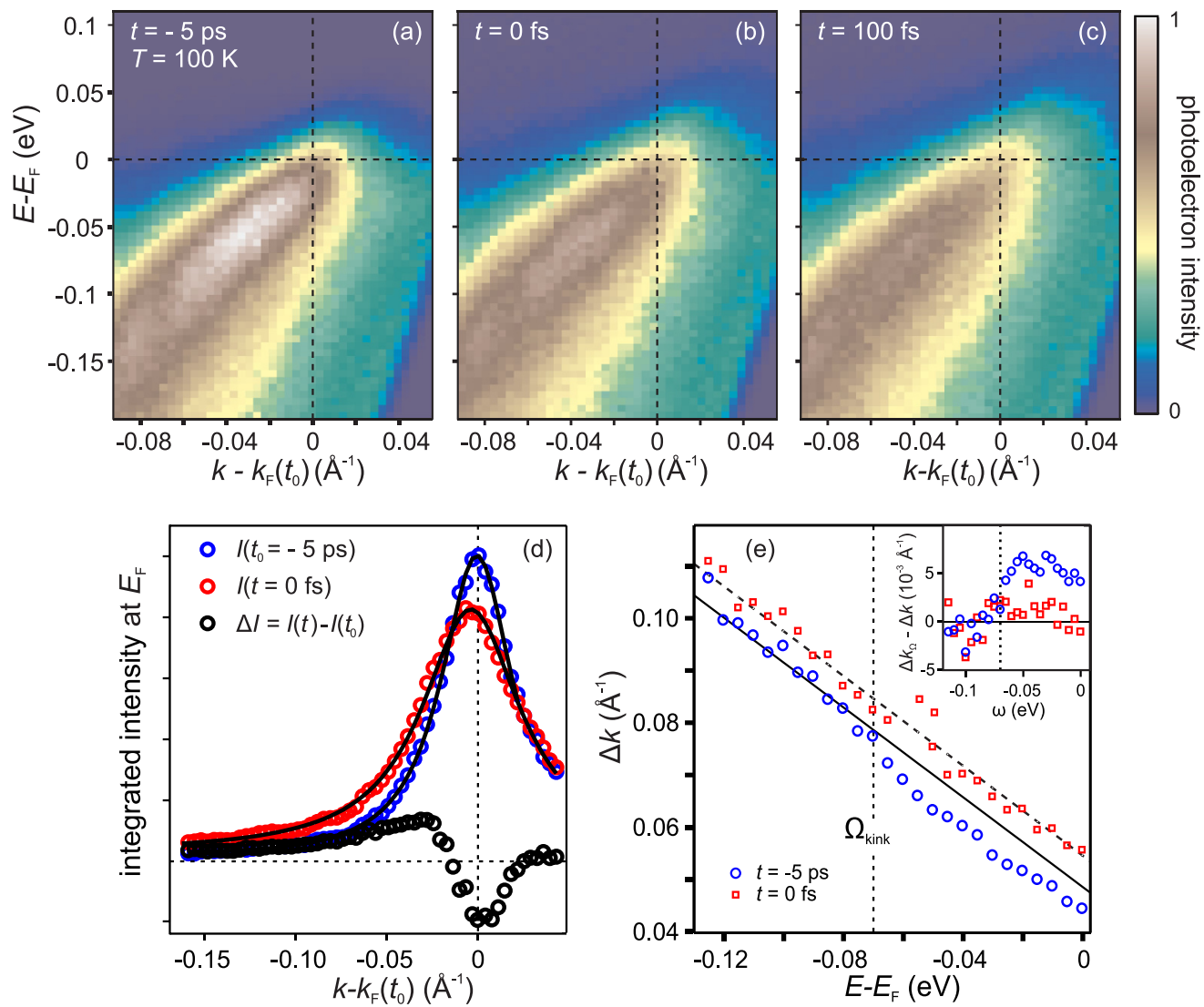

FIG. 1. (Color online) (a)-(c) Photoelectron intensity in a false color representation as a function of electron momentum $k-k_{\mathrm{F}}$ and binding energy with respect to $E_{\mathrm{F}}$ before, during, and after optical excitation, respectively, after applying a Lucy-Richardson deconvolution algorithm (Ref. [19]). The incident pump laser fluence was $300 \mu \mathrm{J} / \mathrm{cm}^{2}$. Panel (d) shows the momentum-dependent intensity at $E_{\mathrm{F}}$ integrated within $\pm 10 \mathrm{meV}$ before and during optical excitation as well as the respective differences. Solid black lines are Lorentzian fits. MDC widths as a function of binding energy are shown in panel (e) for $300 \mu \mathrm{J} / \mathrm{cm}^{2}$ fluence, $t=-5 \mathrm{ps}$ (blue circles), and $t=0$ fs (red squares). The solid line $\left(\Delta k_{\Omega}\right)$ represents a linear least-squares fit to $E-E_{\mathrm{F}}<-70 \mathrm{meV}, t=-5 \mathrm{ps} \mathrm{MDC}$ widths extrapolated to $E_{\mathrm{F}}$. The dashed line has the same slope and is shifted upwards by $6 \mathrm{meV}$. The inset shows $\Delta k_{\Omega}-\Delta k$ versus energy for both delay times to highlight the pump-induced change. 

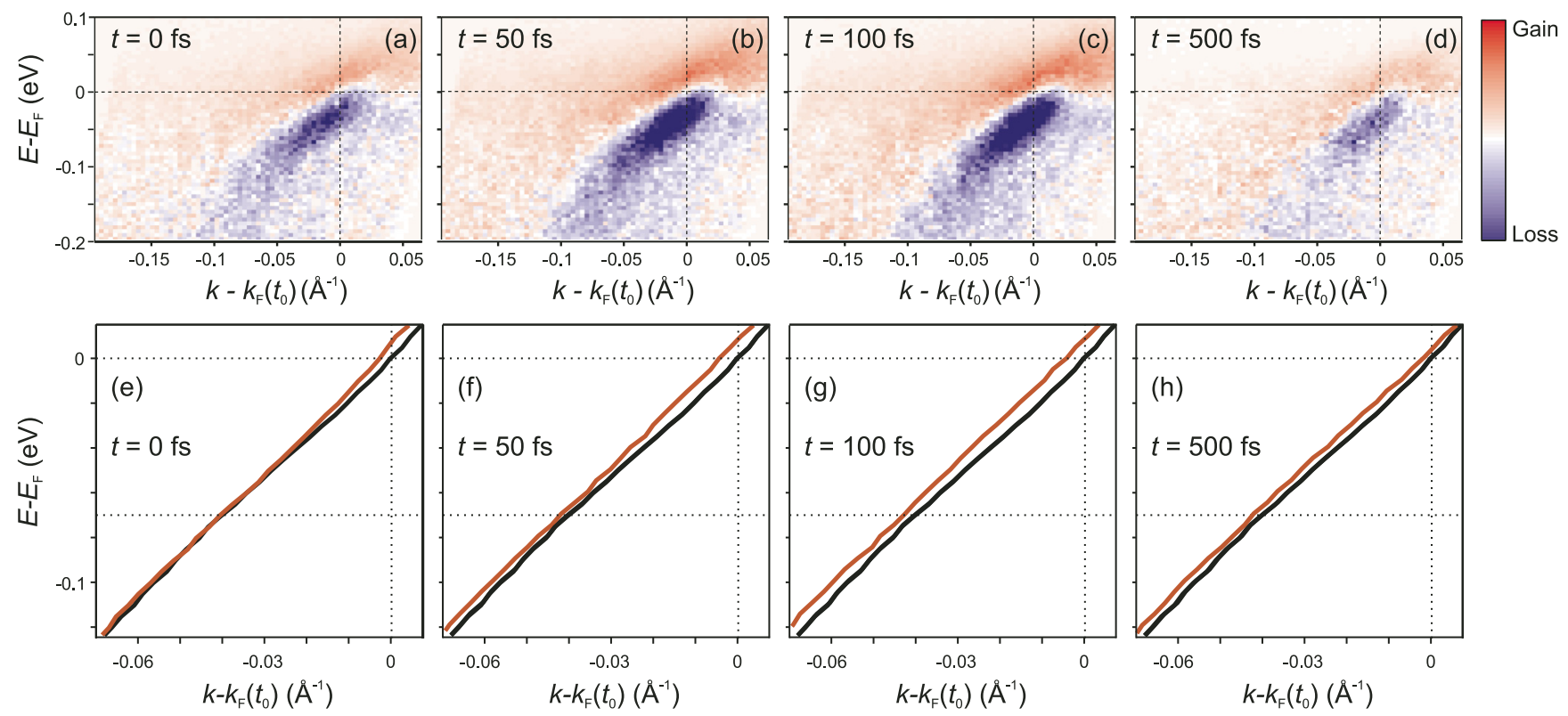

FIG. 2. (Color online) (a)-(d) Laser-pump-induced photoemission intensity difference $I(t)-I\left(t_{0}\right)$ as a function of momentum and energy in a blue-white-red false color representation at indicated delay times. (e)-(h) show the respective dispersions $E(k)$ determined from MDC maxima in red. In black, the dispersion before optical excitation at $t_{0}$ is shown for comparison. Dotted lines through $E_{\mathrm{F}}, k_{\mathrm{F}}$, and $E-E_{\mathrm{F}}=-70 \mathrm{meV}$ are guides to the eye.

difference color maps $\Delta I(E, k, t)=I(E, k, t)-I\left(E, k, t_{0}\right)$, where $t_{0}=-5 \mathrm{ps}$, show that spectral depletion occurs predominantly in the energy range of the coherent quasiparticles at energies between the $70-\mathrm{meV}$ kink and $E_{\mathrm{F}}$. The intensity increase is found to occur in an $E(\boldsymbol{k})$ region adjacent to the unperturbed band and shifted to higher $E$ and smaller $k$. We conclude that there exists a well-defined dispersion in the intermediate excited state, even though the system experiences intense optical excitation generated by the pump-laser field. Comparing intensity difference maps for 0 and $100 \mathrm{fs}$, we recognize a change in the effective mass. An analysis of pump-induced changes in $E(\boldsymbol{k})$ is carried out by fitting MDCs at different delays. $E(\boldsymbol{k})$, given by the MDC peak positions, is shown in Figs. 2(e)-2(h) as a function of energy and momentum and demonstrates our essential findings of a photoinduced reduction in $m^{*}$ and a reduction/shift in $k_{\mathrm{F}}$ [21]. At $t=0, k_{\mathrm{F}}$ and $m^{*}$, which are both determined in the vicinity of $E_{\mathrm{F}}$, are clearly reduced compared to their values before pumping. At the same time, the discontinuity in $\Delta k(E)$ that is observed here at the kink energy for delays before pumping is significantly reduced [Fig. 1(e)] and the coherent weight at low energies is significantly disrupted. At $t=50 \mathrm{fs}$, the shift in $k_{\mathrm{F}}$ has increased, whereas, the dispersion $E(k)$ has partially recovered. At $t=100 \mathrm{fs}$, the modified $k_{\mathrm{F}}$ is maximized, but $m^{*}$ has recovered its equilibrium value. It is not before $t=0.5$ ps that $k_{\mathrm{F}}$ has nearly relaxed to its initial value. Figure 3 shows the relative changes in the effective mass $m_{\%}^{*}(t)=m^{*}(t) / m^{*}\left(t_{0}\right)=v_{\mathrm{F}}\left(t_{0}\right) / v_{\mathrm{F}}(t)$, where $v_{\mathrm{F}}$ is the Fermi velocity, for different pump fluences as a function of time delay.

Within the experimental uncertainties, changes in $m_{\%}^{*}$ are observed for fluences above $35 \mu \mathrm{J} / \mathrm{cm}^{2}$. However, this does not necessarily imply a threshold due to the finite statistics available in the experiments. A remarkable finding is that the ultrafast change essentially occurs instantaneously, without any recognizable time delay compared to the optical excitation and a recovery of the initial value within the cross-correlation width. Therefore, we conclude that the pump-induced change

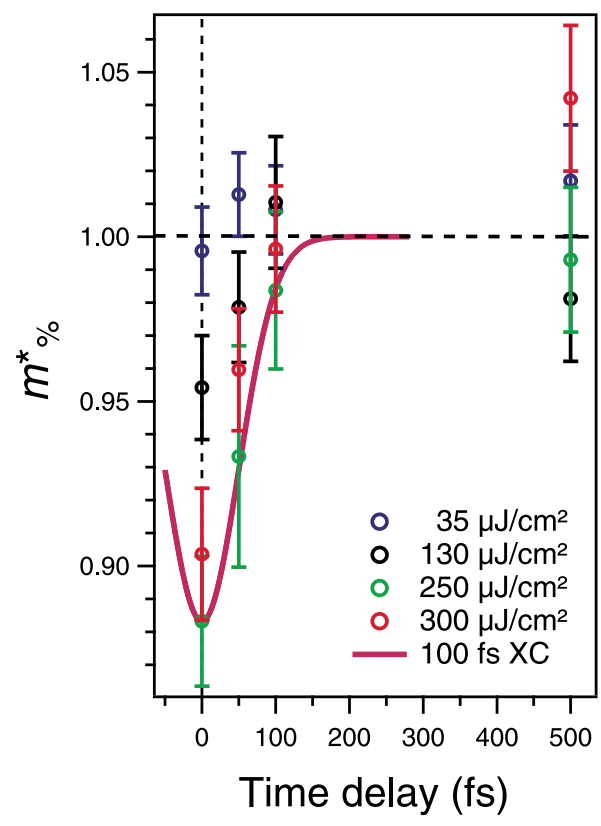

FIG. 3. (Color online) Relative photoinduced change in the effective mass $m_{\%}^{*}$ for different optical excitation densities. The effective mass $m *(t)$ determined at time delay $t$ is normalized to $m *\left(t_{0}\right)$ before optical excitation. This practice eliminates ambiguity associated with the unknown "bare" noninteracting dispersion. The pink line indicates the pump-probe cross correlation of $100-\mathrm{fs}$ temporal XC width. 
in $m^{*}$ is driven by the laser-pump field itself. The fact that the change in $m^{*}$ has already receded for $t=50 \mathrm{fs}$ sets an upper limit for the response time of $m^{*}$. This observation raises the question of which interactions can be considered to be responsible for the pump-induced modification of $m^{*}$. Optical excitation will heat the lattice as described, e.g., by the two-temperature model or more sophisticated considerations $[14,22,23]$. Such heating, which is characterized by a well-defined lattice temperature including a thermalized distribution of excitations shared among optical and acoustic phonons, requires time scales of several picoseconds to build up and, as such, can be excluded here. Optical excitation could, however, involve ultrafast coupling to high-energy optical phonons. Such vibrations have been proposed to be responsible for the 70-meV kink [2]. We exclude this scenario here because the decay into acoustic modes subsequent to excitation of such optical phonons would occur on the much slower picosecond time scale mentioned above [23]. This is corroborated by the fluence dependence of the effective-mass change (Fig. 3). In the case of relaxation of the excited state through phonons, an increase in pump fluence would inevitably result in stronger heating, leading to a more pronounced reminiscence of pumping at later delays. However, within error bars, no effective-mass change is found at 100 fs for all fluences. Furthermore, it is unclear how such optically excited high-frequency phonon modes might couple directly to the Fermi surface where the dispersion changes most radically.

Our data in Figs. 2(e) and 2(f) show that the change in $k$ at $70 \mathrm{meV}$ begins to appear at 50-fs delay and not at 0 fs when the mass effect is at its apex. To explain the observed reduction in $m^{*}$, we, therefore, favor the dissolution of electronic correlations by the optical pump field as suggested by Sentef et al. [24]. The kink is weakened because incoherent electronic states cannot couple coherently at a well-defined energy. That the kink is transiently wiped out by disrupting the coherence of states with energy too low to excite a putative bosonic mode at equilibrium, rather than by decoupling high-energy electronic states from their environment, is consistent with broadening effects at all energies observed in the present experiment possessing an electronic origin. According to Fig. 1(e), the only difference between states above and below the kink energy at $t=0$ is that the latter are dressed by externally applied rather than native electronic excitation. The lack of renormalization of the band velocity above the kink energy with decreasing discontinuity in the MDC widths at the peak of the excitation process is also inconsistent with a bosonic origin for the kink. We, therefore, conclude that underlying electronic correlations must also play a role in the formation of the $70-\mathrm{meV}$ kink in equilibrium.

Finally, we return to the observation of a pump-induced change in $k_{\mathrm{F}}$. We have analyzed these changes for different pump fluences and time delays and have estimated the resultant changes in hole doping $\delta x$ by modeling the Fermi surface of cuprates following the Yang-Rice-Zhang ansatz [25]. As depicted in Fig. 4(a), we find a continuous increase in the $k_{\mathrm{F}}$ shift for larger fluences for all time delays studied. We determined the Fermi velocity $v_{\mathrm{F}}$ from the slope of a leastsquares linear fit $\left(\omega-\omega_{0}=v_{\mathrm{F}}\left[k-k_{\mathrm{F}}\right]\right)$ between $\hbar \omega=-25$ and $5 \mathrm{meV}$. To improve the accuracy in $k_{\mathrm{F}}$, we determine it from $k_{\mathrm{F}}=-\omega_{0} / v_{\mathrm{F}}$, where $\omega_{0}$ is the abscissa intercept given
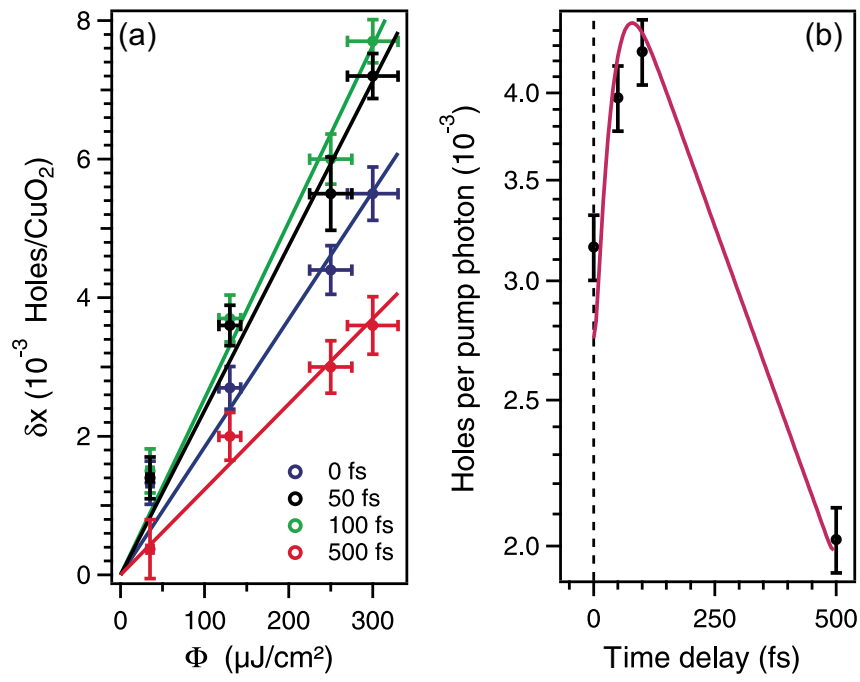

FIG. 4. (Color online) Effective photoinduced hole doping $\delta x$ (a) as a function of fluence $\Phi$ and (b) as a function of delay time after normalizing to the numbers of absorbed pump photons per unit cell. The analysis is based on the time-dependent shift in $k_{\mathrm{F}}$ (see Figs. 1 and 2). The determination of the number of holes per $\mathrm{CuO}_{2}$ unit cell shown in (a) assumes the model of the Fermi surface in cuprates by Yang et al. [25]. The lines representing least-squares linear fits to the fluence dependent $\delta x$ assume a vanishing photodoping for an absent excitation. The points in (b) are derived from the slopes of these linear fits. The pink line in (b) guides the eye and is an exemplary single exponential decay function convoluted with the cross-correlation width for pump and probe laser pulses. Errors in $v_{\mathrm{F}}$ and $k_{\mathrm{F}}$ result from the least-squares fits.

by the fit. The time evolution of the number of carriers excited per photon, shown in Fig. 4(b), is, however, different from that of $m^{*}$. The maximum in $\delta x$ occurs at a finite time delay of 50-100 fs, indicating that secondary electronic excitations, to which initial excitations decay [26], induce the $k_{\mathrm{F}}$ change. This is plausible considering the effect's decay within 1 ps because, on this time scale, the coupling of electrons to phonons has time to proceed [22,23]. Such photodoping has been reported before for topological insulators and Fe-based superconductors [27-29]. However, its quantification relied on the analysis of the chemical potential $\mu$. Here, we are able to trace the change in $k_{\mathrm{F}}$, which is proportional to carrier density and, consequently, to estimate the absolute number of photoexcited holes per pump photon. To explain our observation, we note that the pump process results in the excitation of electron and hole distributions. However, the known electron-hole asymmetry in the cuprates [30] results in a difference in screening of the electrons and holes leading to an increased effective hole population, which is then compensated by a rearrangement of $k_{\mathrm{F}}$. The photodoping of a doped Mott insulator, therefore, appears to more closely resemble that of a semiconductor than a true metal.

\section{CONCLUSIONS}

We have reported two novel phenomena driven by laserinduced changes in electronic structure. The first one is a transient photoinduced change in $m^{*}$ in a solid, which 
occurs during the presence of the 100-fs time resolution and suggests a primarily light-induced change in the electronic interaction underlying the dressing of the electronic quasiparticles. Second, we demonstrate photodoping in the investigated optimally doped cuprate up to a hole concentration of about $10^{-2}$ holes $/ \mathrm{CuO}_{2}$, which is delayed with respect to the effective-mass change and is consistent with relaxation of the excited state by secondary electrons. The different time scales at which these effects appear indicate that transient changes in doping and $m^{*}$ constitute intertwined, yet microscopically distinct, phenomena. Finally, considering the fundamental importance of doping in devices, we conclude by noting that a controlled modification of transient doping appears to be promising for future optoelectronic devices. The investigation of a femtosecond time-scale photoinduced transition between an insulating to a metallic or even to a superconducting state will, therefore, be of paramount technological as well as scientific interest, devices based on such effects possibly possessing switching bandwidths on the order of many hundreds of gigahertz.

\section{ACKNOWLEDGMENTS}

We acknowledge funding from the Deutsche Forschungsgemeinschaft through Sfb 616 and SPP 1458, from the Mercator Research Center Ruhr through Grant No. PR-2011-0003, and from the European Union within the seventh Framework Program under Grant No. 280555 (GO FAST). The work at Brookhaven Laboratory was supported by the Center for Emergent Superconductivity (CES), an Energy Frontier Research Center funded by the US DOE, Office of Basic Energy Sciences. We would like to acknowledge useful conversations with T. M. Rice, M. Sentef, and A. F. Kemper.
[1] T. Valla et al., Science 285, 2110 (1999).

[2] A. Lanzara et al., Nature (London) 412, 510 (2001).

[3] X. J. Zhou et al., Nature (London) 423, 398 (2003).

[4] X. J. Zhou et al., Phys. Rev. Lett. 95, 117001 (2005).

[5] A. Kaminski, M. Randeria, J. C. Campuzano, M. R. Norman, H. Fretwell, J. Mesot, T. Sato, T. Takahashi, and K. Kadowaki, Phys. Rev. Lett. 86, 1070 (2001).

[6] P. D. Johnson et al., Phys. Rev. Lett. 87, 177007 (2001).

[7] S. V. Borisenko et al., Phys. Rev. Lett. 96, 117004 (2006).

[8] K. Byczuk et al., Nat. Phys. 3, 168 (2007).

[9] Dynamics at Solid State Surfaces and Interfaces: Volume 1Current Developments, edited by U. Bovensiepen, H. Petek, and M. Wolf (Wiley-VCH, Weinheim, 2010).

[10] J. Demsar, B. Podobnik, V. V. Kabanov, T. Wolf, and D. Mihailovic, Phys. Rev. Lett. 82, 4918 (1999).

[11] N. Gedik, P. Blake, R. C. Spitzer, and J. Orenstein, Phys. Rev. B 70, 014504 (2004).

[12] R. A. Kaindl, M. A. Carnaham, and D. S. Chemla, Phys. Rev. B 72, 060510(R) (2005).

[13] S. Dal Conte et al., Science 335, 1600 (2012).

[14] C. Gadermaier, A. S. Alexandrov, V. V. Kabanov, P. Kusar, T. Mertelj, X. Yao, C. Manzoni, D. Brida, G. Cerullo, and D. Mihailovic, Phys. Rev. Lett. 105, 257001 (2010).

[15] R. Cortés, L. Rettig, Y. Yoshida, H. Eisaki, M. Wolf, and U. Bovensiepen, Phys. Rev. Lett. 107, 097002 (2011).

[16] L. Rettig, R. Cortés, S. Thirupathaiah, P. Gegenwart, H. S. Jeevan, M. Wolf, J. Fink, and U. Bovensiepen, Phys. Rev. Lett. 108, 097002 (2012).

[17] C. L. Smallwood et al., Science 336, 1137 (2012).

[18] P. S. Kirchmann, L. Rettig, D. Nandi, U. Lipowski, M. Wolf, and U. Bovensiepen, Appl. Phys. A 91, 211 (2008).
[19] J. D. Rameau, H.-B. Yang, and P. D. Johnson, J. Electron Spectrosc. Relat. Phenom. 181, 35 (2010).

[20] Original data qualitatively show the same effects.

[21] For each $(t, \Phi)$, the $(k, \omega)$ cut identified in Fig. 1 is deconvolved using the Lucy-Richardson deconvolution method. Every cut is treated identically by running three deconvolution iterations with $\Delta k=0.002 \AA^{-1}$ and $\Delta \omega=55 \mathrm{meV}$. Each deconvolved spectrum is fit by momentum distribution curves at each binding energy. The fitting was performed using Lorentzians on a linear background. The peak positions extracted from this procedure constitute the dispersions shown in Figs. 2(d)-2(g). The $\chi^{2}$ fit errors to the peak positions are generally on the order of the line thickness.

[22] P. B. Allen, Phys. Rev. Lett. 59, 1460 (1987).

[23] L. Perfetti, P. A. Loukakos, M. Lisowski, U. Bovensiepen, H. Eisaki, and M. Wolf, Phys. Rev. Lett. 99, 197001 (2007).

[24] M. Sentef, A. F. Kemper, B. Moritz, J. K. Freericks, Z.-X. Shen, and T. P. Devereaux, Phys. Rev. X 3, 041033 (2013).

[25] K.-Y. Yang, T. M. Rice, and F.-C. Zhang, Phys. Rev. B 73, 174501 (2006).

[26] W. Nessler, S. Ogawa, H. Nagano, H. Petek, J. Shimoyama, Y. Nakayama, and K. Kishio, Phys. Rev. Lett. 81, 4480 (1998).

[27] Y. H. Wang, D. Hsieh, E. J. Sie, H. Steinberg, D. R. Gardner, Y. S. Lee, P. Jarillo-Herrero, and N. Gedik, Phys. Rev. Lett. 109, 127401 (2012).

[28] A. Crepaldi, B. Ressel, F. Cilento, M. Zacchigna, C. Grazioli, H. Berger, Ph. Bugnon, K. Kern, M. Grioni, and F. Parmigiani, Phys. Rev. B 86, 205133 (2012).

[29] I. Avigo et al., J. Phys.: Condens. Matter 25, 094003 (2013).

[30] H. B. Yang et al., Nature (London) 456, 77 (2008). 